\title{
Filipinas: Los 'Campeones' promueven la elaboración de políticas basadas en investigación
}

Frontiers in Reproductive Health

Follow this and additional works at: https://knowledgecommons.popcouncil.org/departments_sbsr-rh How does access to this work benefit you? Let us know!

\section{Recommended Citation}

"Filipinas: Los 'Campeones' promueven la elaboración de políticas basadas en investigación," FRONTERAS Resumen de Investigación Operativa. Ciudad de México: Population Council, 2003. 


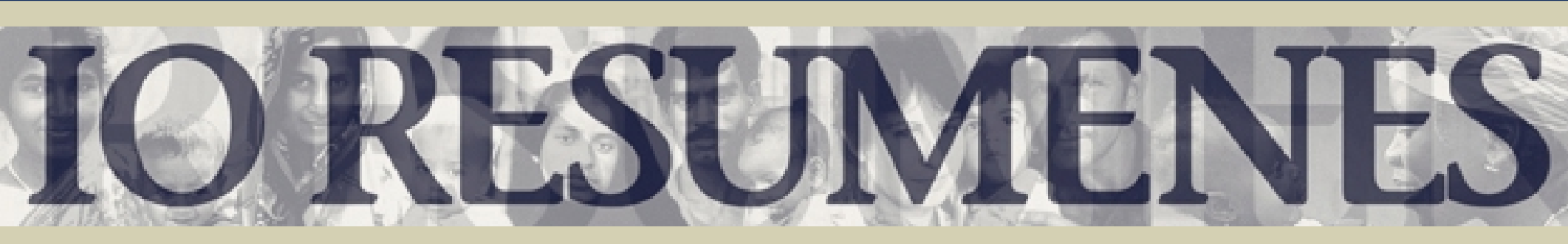

\section{Filipinas Utilización}

\author{
Investigación \\ Operativa
}

Resumen 20

\section{Los "Campeones" promueven la elaboración de políticas basadas en investigación}

\author{
Los "Equipos de Campeones de las Políticas" tuvieron éxito al promover el \\ uso de un sistema de información evaluado, que sirve para identificar a las \\ clientas con necesidades insatisfechas de planificación familiar. Esta \\ estrategia fue usada por varias organizaciones, que piensan extender el uso \\ de este sistema de información como herramienta gerencial.
}

\section{Antecedentes}

En septiembre de 2000, el Population Council puso en marcha un proyecto que duró 9 meses, con el fin de probar la efectividad de "Equipos de Campeones" que promueven el uso de resultados de investigación operativa para formular políticas y programas de salud reproductiva en Filipinas. La estrategia está basada en evidencia que muestra que los gerentes de programas de salud descentralizados tienen poco conocimiento sobre salud reproductiva y muy rara vez utilizan los resultados de investigación para tomar decisiones.

El proyecto estuvo enfocado en el uso de un sistema de información y monitoreo de las comunidades (CBMIS, por sus siglas en inglés), el cual fue probado mediante investigación operativa. El CBMIS ayuda a los proveedores de salud a identificar y responder a las necesidades insatisfechas de planificación familiar de las mujeres, y les facilita a las mismas el acceso a los servicios de salud. El estudio mostró que este sistema centrado en el cliente es muy útil para que parteras, trabajadoras de salud comunitaria y supervisores locales hagan sus planes de trabajo enfocando los esfuerzos a las mayores necesidades. El CBMIS contribuyó también a mejorar la comunicación entre proveedores y clientes. Con estos resultados, el director provincial de salud en Davao del Norte utilizó los fondos existentes para ampliar el sistema de información a toda la provincia, con lo cual logró incrementar la cobertura de 130,000 a más de 700,000 personas.
Para que más programas pudieran utilizar este sistema de información, los gerentes formaron equipos compuestos por cinco o seis personas respetadas y comprometidas con sus comunidades, en cuatro regiones de Mindanao. Los integrantes de los equipos provenían de instituciones gubernamentales, no gubernamentales, académicas y de medios de comunicación. Los "Equipos de Campeones" recibieron tres días de orientación para convertirse en promotores de políticas. Durante la orientación, se demostró el uso de la CBMIS como herramienta para identificar las necesidades insatisfechas de salud de la población, y se abordaron otros temas para ayudar a los promotores del sistema de información a hacer sus presentaciones de manera profesional, poniendo atención en el diseño de mensajes dirigidos a públicos específicos (en este caso, coordinadores de proyectos) con el fin de influir en las políticas locales y lograr cambios.

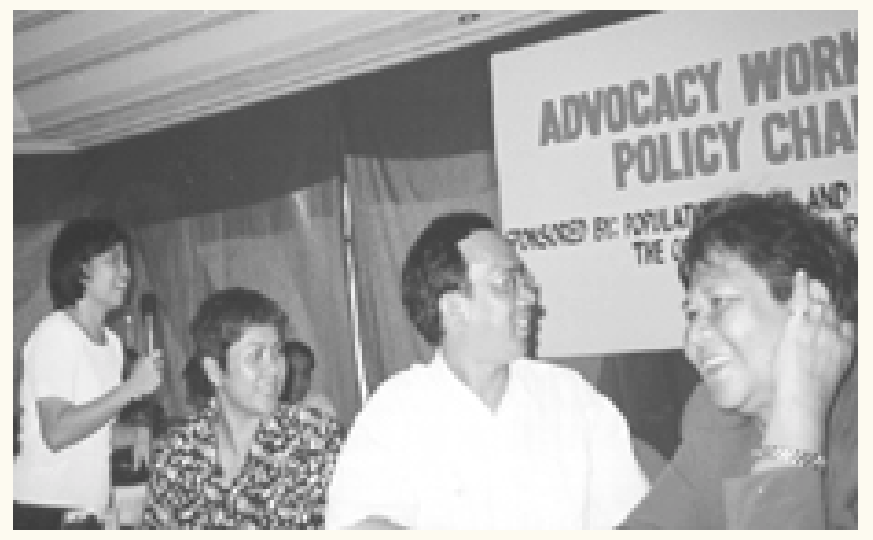




\section{Hallazgos}

- La estrategia de "Campeones" fue efectiva para convencer a los coordinadores de utilizar el CBMIS para mejorar los servicios de planificación familiar. En dos de los cuatro proyectos que se llevaron a cabo, los coordinadores realizaron pruebas piloto para adoptar el CBMIS. En dos ciudades donde ya funcionaba el CBMIS, los coordinadores se comprometieron a continuar usándolo. En otra región, los coordinadores organizaron "Equipos de Campeones" locales para continuar los esfuerzos iniciados por el equipo regional. Un alcalde en la misma región creó un equipo de trabajo sobre género, salud y planificación familiar, que fungirá como secretaría del CBMIS.

\section{"Sentí que soy importante para alcanzar las metas de salud reproductiva en el país. Como promotor de políticas, me di cuenta de lo grande que es mi responsabilidad". -Un promotor de políticas.}

- La disponibilidad de una masa crítica de hallazgos de investigación, relevantes a nivel local, contribuyó al éxito del proyecto. Por ejemplo, la investigación operativa había mostrado que el CBMIS era (1) efectivo para identificar necesidades no satisfechas de salud; (2) más efectivo que otros sistemas de recolección de datos que se estaban utilizando, y (3) compatible con los requerimientos locales de información. La fuerza y calidad de la evidencia favorable propició la adopción del sistema en zonas donde todavía no se había instalado.

- Durante la promoción y el cabildeo se hizo una presentación sencilla del problema, dirigida a los tomadores de decisiones más importantes. Los cuatro equipos de promotores de políticas utilizaron al menos tres estrategias para facilitar la utilización: coordinarse con las personas importantes; darles información sobre el problema de las necesidades insatisfechas en salud, y hacerlos participar en la formulación de planes para utilizar el sistema de información.

\section{Utilización}

- Este modelo de "Equipo de Campeones" fue adoptado por la Comisión de Población, el organismo nacional encargado de coordinar, monitorear y elaborar las políticas del Programa Nacional de Población. La meta es institucionalizar el CBMIS para integrar los servicios de planificación familiar con otros servicios de salud, como son la atención a la salud materna e infantil y la detección de cáncer cervical utilizando la prueba del Papanicolaou.

- Otros proyectos de USAID en Filipinas, en especial uno conducido por Management Sciences for Health, han utilizado los resultados del proyecto de CBMIS para mejorar la orientación que se proporciona a las clientas de planificación familiar.

Las lecciones más importantes del proyecto son:

- Los coordinadores de programas están abiertos a nuevos enfoques para mejorar los servicios

- No existe un enfoque único para lograr un cambio en las políticas, pero se debe iniciar con una presentación clara del problema, dirigida a las personas involucradas en la toma de decisiones

- Hay que mostrar la evidencia de que los resultados son confiables

- El apoyo institucional es fundamental para la asimilación de resultados y su posterior utilización

- Es necesario involucrar a las personas influyentes desde el principio para asegurar su participación más adelante

- La creatividad y la colaboración son la clave para asegurar los recursos para implementar la estrategia a mayor escala

- Los gerentes que quieren que se haga un mayor uso de los resultados de investigaciones para tomar decisiones sobre políticas y programas podrían usar el modelo de promotores "Campeones" para lograrlo. Se vuelve a recomendar que se les capacite en el uso del sistema de información y en la comunicación interpersonal efectiva para que presenten de manera clara el problema, los resultados de investigación y sus implicaciones normativas.

Enero 2003

\section{Fuentes:}

Masulit, Saniata, Marilou Costello y Sahar Hegazi. 2001. "Influencing reproductive health policy and programs in the Philippines: Implementing an advocacy model for utilization of operations research [Implementación de un modelo de promoción para la utilización de la investigación operativa para influir sobre las políticas y programas de salud reproductiva en Filipinas]," FRONTIERS Final Report. Washington, DC: Population Council. Disponible en nuestro portal: http://www.popcouncil.org/pdfs/frontiers/FR FinalReports/ Philippines Influencing Policy.pdf o por correo electrónico: frontiers@pcdc.org

Jain, Anrudh, Saumya RamaRao, Marilou Costello, Marlina Lacuesta y Napoleón Amoyen. 2002. “Learning about clients' needs: Family planning field workers in the Phlippines [Conocer las necesidades de las clientas: trabajadores de planificación familiar en Filipinas]," en Haberland, N. y D. Measham (eds.), Responding to Cairo: Case studies of changing practice in reproductive health and family planning [Respondiendo a Cairo: Estudios de caso sobre el cambio en las prácticas de salud reproductiva y planificación familiar]. Nueva York: Population Council. Disponible en nuestro portal: http://www.popcouncil.org/cairocasestudies/index.html o por correo electrónico: frontiers@pcdc.org

Este proyecto se llevó a cabo con el apoyo de la AGENCIA DE LOS ESTADOS UNIDOS PARA EL DESARROLLO INTERNACIONAL (USAID) bajo el Acuerdo de Cooperación No. HRN-A-00-98-00012-00.

\section{Population Council}

